Received: 16 January 2017

Accepted: 11 December 2017

Published online: 22 December 2017

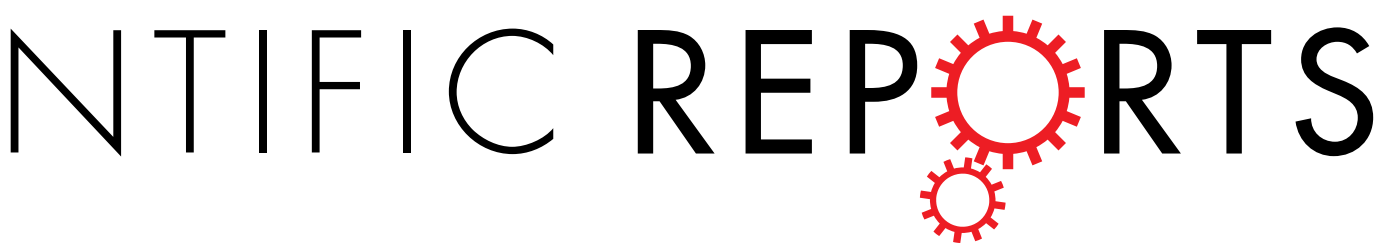

\title{
Intravitreal injections of anti-VEGF agents and antibiotic prophylaxis for endophthalmitis: A systematic review and meta-analysis
}

Manuel F. Bande ${ }^{1}$, Raquel Mansilla ${ }^{1}$, María P. Pata ${ }^{2}$, Maribel Fernández ${ }^{1}$, María José BlancoTeijeiro ${ }^{1}$, Antonio Piñeiro ${ }^{1} \&$ Francisco Gómez-Ulla ${ }^{1}$

We performed a systematic review and meta-analysis to determine whether the use of local antibiotics is a beneficial prophylactic treatment for endophthalmitis in patients treated with anti-VEGF agents. We searched the MEDLINE and EMBASE databases, and the Cochrane Library over the period January 2007 to December 2016. The search terms used included "Endophthalmitis", "Antibiotic" and "Intravitreal injection". Studies in which the patients were treated exclusively with intravitreal injections of antiVEGF were selected. Eight studies fit the inclusion criteria, which included a total of 276,774 injections; $109,178(39.45 \%)$ were associated with the use of antibiotics and $114,821(60.55 \%)$ were not associated with the use of antibiotics. Our meta-analysis indicated a significant risk for endophthalmitis that was 1.70 times greater with the use of antibiotics than that without antibiotics, with a confidence interval of 1.08 to $2.66(p=0.02)$. A meta-regression indicated that the location (operating rooms versus outpatient clinics) of injection did not have a significant effect on the incidence of endophthalmitis. The prophylactic use of antibiotics when administering anti-VEGF intravitreal injections may contribute to a greater incidence of endophthalmitis. This finding, in addition to reducing costs, would eliminate a treatment that has been shown to be unnecessary and even harmful to patients.

Intravitreal anti-vascular endothelial growth factor (VEGF) therapy is frequently used as one of the main treatments for many retinal pathologies, including age-related macular degeneration (exudative) ${ }^{1}$, diabetic macular edema $^{1,2}$ and retinal vein occlusion ${ }^{3}$. The use of anti-VEGF agents has increased in recent years and now constitutes one of the most common procedures in ophthalmology. It is projected that this trend will continue for the next few years. Although the risk of injection-associated endophthalmitis is low (approximately 1 in 3000 injections $)^{4}$, the visual morbidity of this complication is devastating ${ }^{5}$.

The only preventive measure with some consensus regarding its effectiveness is the application of povidone-iodine to the ocular surface prior to injection, which is the only effective prophylactic measure supported by clinical trials ${ }^{6}$. Other measures, such as the use of gloves, a blepharostat or masks and the setting where injection was given (operating rooms or outpatient clinics), remain controversial ${ }^{7,8}$. The use of antibiotics as a method of prophylaxis is part of the normal practice of intraocular procedures. However, recent studies have shown that antibiotics offer no protection against the risk of developing endophthalmitis once anti-VEGF injections have been administered, and, in some cases, the rates of endophthalmitis are actually higher in groups using antibiotics ${ }^{9,10}$. Although the abandonment of antibiotics before and after intravitreal administration of anti-VEGF appears to be the current trend, the use of antibiotics remains part of the normal procedure for a significant percentage of practitioners ${ }^{11,12}$.

Currently, no consensus exists regarding the benefits of antibiotic prophylaxis of endophthalmitis after anti-VEGF injections. To clarify this controversy, we present a meta-analysis to evaluate the incidence of endophthalmitis after treatment with anti-VEGF agents associated with 1) use of topical antibiotics and 2) the setting where the injection is performed. 


\begin{tabular}{|c|c|c|c|c|c|c|c|c|}
\hline Study & Place & Timing (AB) & Injections & Endoph & Inject. $\mathrm{AB}$ & Endoph. AB & Inject. no $\mathbf{A B}$ & Endoph. no AB \\
\hline Falavarjani et al. ${ }^{33}$ & 2 & 2 & 5,901 & 6 & 3,975 & 6 & 1,926 & 0 \\
\hline Cheung et al. ${ }^{* 34}$ & 1 & 2 & 14,960 & 7 & 10,061 & 6 & 4,899 & 1 \\
\hline Fineman et al. ${ }^{8}$ & 1 & 2 & 10,164 & 3 & 7,415 & 2 & 2,749 & 1 \\
\hline Mason et al..$^{35}$ & 1 & 2 & 5,233 & 1 & 2,617 & 1 & 2,616 & 0 \\
\hline Park et al. ${ }^{36}$ & 1 & 1 & 16,186 & 2 & 8,078 & 0 & 8,108 & 2 \\
\hline Falavarjani et al..$^{37}$ & 2 & 2 & 8,037 & 1 & 2,771 & 1 & 5,266 & 0 \\
\hline Storey et al..$^{38}$ & 1 & 3 & 147,479 & 52 & 57,654 & 28 & 89,825 & 24 \\
\hline Li et al. $^{* 39}$ & 1 & 3 & 68,814 & 15 & 16,607 & 4 & 52,207 & 11 \\
\hline
\end{tabular}

Table 1. Summary of studies selected for inclusion in the meta-analysis. Codes: Place: $1=$ Outpatient clinics, $2=$ Operating room; AB: $1=$ Pre-injection, $2=$ Post-injection, $3=$ Pre- and post-injection, $4=$ No AB. Abbreviations: Injec.: injections, Endoph.: endophthalmitis, and AB: antibiotic. *Extra information provided by the authors.

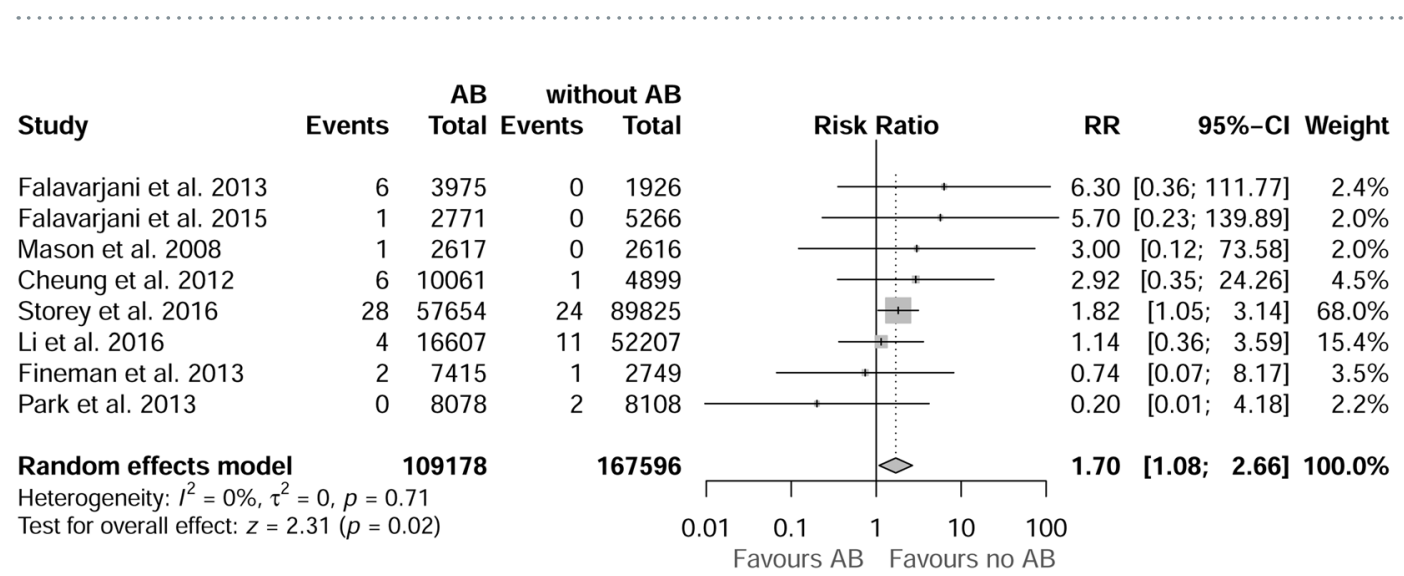

Figure 1. Forest plot with relative risk (RR) estimates of each study and the combined RR (represented as a rhombus), including the $95 \%$ confidence intervals and the weights assigned to each study.

\section{Results}

An original search produced 779 possible articles. Removal of duplicates and application of selection criteria reduced the number of articles comparing two groups to eight. As shown in these publications, the proportion of cases of endophthalmitis among the total patients analyzed in each publication was very low (0.035\%), with values ranging from $0.012 \%$ to $0.100 \%$. In cases of endophthalmitis with and without antibiotic prophylaxis, staphylococcus and streptococcus were the most frequent microorganisms identified in culture-proven endophthalmitis. In six of the studies, the injections were administered in an operating room, and in the two remaining studies, the injections were administered in outpatient clinics. Table 1.

Relationship between antibiotic therapy and endophthalmitis. To perform this meta-analysis, we selected publications that indicated both the number of anti-VEGF injections associated with the use of antibiotics as well as the number of injections not associated with the use of antibiotics. The studies included in this analysis were characterized by homogeneity regarding the type of healthcare professional that administered the injections (ophthalmologist) and the use of povidone in all cases.

Eight studies were selected, involving a total of 276,774 injections, with 109,178 (39.45\%) injections associated with the use of antibiotics and 167,596 (60.55\%) injections not associated with the use of antibiotics. The relative risk (RR) of endophthalmitis was 1.70 times greater with the use of antibiotics than without antibiotics, with a confidence interval of 1.08 to 2.66 (the number of cases of endophthalmitis in $\mathrm{AB}$ group and non-AB was 48 and 39 , respectively). Therefore, the use of prophylactic antibiotics is associated with a higher incidence of endophthalmitis $(\mathrm{z}=2.31, \mathrm{p}=0.02$ ). The forest plot (Fig. 1 ) shows the RR and $95 \%$ confidence intervals for each of the studies and the combined RR obtained from the random effects model.

No heterogeneity was observed in the $\mathrm{I}^{2}$ value, which indicates a null-low heterogeneity $\left(\mathrm{I}^{2}=0 \%(0 \%, 31 \%)\right)$, or with the test of heterogeneity $(\mathrm{Q}$ (degrees of freedom $)=4.52(7), \mathrm{p}=0.717)$. Both the graphical analysis (Figure S1) and the regression test $(\mathrm{t}=0.007, \mathrm{p}$-value $=0.994)$ indicated the absence of publication bias. With respect to the sensitivity analysis, after excluding the study by Story et al., which had a sample size much greater than the remaining sample, the RR decreased to $1.49(0.7664,3.3016)$, but heterogeneity increased substantially (I $2=0 \%-61 \%)$. The number of cases of endophthalmitis in $\mathrm{AB}$ group and non-AB was 20 and 15 respectively, when Storey et al. was excluded from the analysis. 
Meta-regression analysis of the effect of the setting where injection was given. Meta-regression was performed to assess whether the setting where injection was administered (operating room versus outpatient clinics) influenced the incidence of endophthalmitis associated with antibiotic treatment. The analysis results $(\mathrm{z}=1.165, \mathrm{p}=0.243)$ suggest that the setting where the injection was administered had no significant effect on the incidence of endophthalmitis associated with antibiotics.

Moreover, the results of the various meta-regressions showed no significant effect of any of the following variables on the incidence of endophthalmitis associated with antibiotic treatment: (a) the time at which the antibiotic was administered (pre- versus post-injection antibiotics): $Q M=2 ; 42, p=0.298$ and (b) the causative microorganism: $\mathrm{QM}=0 ; 02, \mathrm{p}=0.905$.

\section{Discussion}

This study presents a meta-analysis that indicates an increased risk of endophthalmitis in patients treated with intravitreal anti-VEGF agents who were also treated with prophylactic topical antibiotics. Our analysis also revealed no significant differences in the risk of developing endophthalmitis for patients who received injections in the operating rooms versus those who received injections in outpatient clinics.

We have also attempted to conduct this study in as rigorous a manner as possible and took extra precautions to control for heterogeneity across studies and publication bias to achieve maximum reliability of the results. The main assumption casting doubt on the validity of the meta-analysis is the lack of significant variations in the procedures for various valued tests. Another factor that adds value to our statistical analysis is the importance of the study by Storey et al. to our conclusions. Increasing the sample size is very important when studying diseases with low incidence such as endophthalmitis

Recent studies have indicated that the use of topical antibiotics could increase resistance to some antibiotics by affecting the conjunctival and nasopharyngeal flora. Moreover, increasing the proportion of resistant bacteria on the ocular surface increases the risk of developing antibiotic-resistant infections that are difficult to treat ${ }^{13}$. Several possibilities could explain the trend of increasing antibiotic resistance. Patients receiving anti-VEGF intravitreal injection therapy for retinal diseases often require repeated doses for long periods of time. Our data confirm that short courses of topical antibiotics affect the patterns of resistance of periocular flora. Fluoroquinolones are the most commonly used post-injection prophylactic antibiotics in patients due to their broad spectrum and high penetration. Several studies have demonstrated substantial levels of resistance to third- and fourth-generation fluoroquinolones, as well as multi-drug resistance in patients treated with topical antibiotics after multiple intravitreal injections ${ }^{14,15}$. On the other hand, it would be necessary to take into account the cost saving that may result from the non-instillation of antibiotic eye drops in patients undergoing this type of treatment.

Among other factors that could influence the risk of developing intraocular infection, consideration of the location of treatment administration is essential. A study based on an analysis of 12,249 injections performed by the same surgeon showed significantly lower levels of endophthalmitis when the injection was performed in the operating room instead of outpatient clinics ${ }^{16}$. By contrast, another study showed that the rate of endophthalmitis after an intravitreal injection was low regardless of whether the procedure was performed in outpatient clinics or in an operating room ( 0.035 versus $0.065 \%$, respectively $)^{17}$. The results of our meta-analysis support this finding.

Recently, Dutheil et al. ${ }^{18}$ presented a similar meta-analysis based on various types of intravitreal injections, including triamcinolone, dexamethasone and perfluoropropane (CF8). The goal of our work was to improve the protocol for the use of anti-VEGF drugs. The inclusion of corticosteroids in an analysis of the rates of endophthalmitis in intravitreal injections may interfere with this objective. Some studies support the idea that the risk of endophthalmitis is not equal among the possible options for intravitreal treatment. Triamcinolone presents a significant increase in the OR of 6.92 for endophthalmitis compared to anti-VEGF agents ${ }^{19}$. This result is partly explained by the immunosuppression that corticosteroids produce in the ocular microenvironment ${ }^{20}$.

However, it is necessary to consider of the following limitations in our study: methodological limitations due to a lack of access to patient data-level statistics, sensitivity bias and considering only those manuscripts published in English. All eight studies included in our analysis were retrospective reviews; they were all case-control studies rather than randomized controlled trials due to the fact that the evaluated randomized trials did not met inclusion criteria. Some of case-control studies were carried out in different periods of time, which may have carried changes in hospital prophylaxis procedures; those aspects could not be considered when evaluating the data. Furthermore, none of the studies provided differentiation of positive cultures between groups with/without antibiotic use; therefore, this variable could not be analyzed in our study. However, the data from the eight studies were sufficient to assess the duration of antibiotic treatment and the RR. Therefore, although our findings seem conclusive, these limitations suggest that the results of this study should be interpreted with caution.

We ultimately believe that the decision to use antibiotics in the prophylactic period depends on individual ophthalmologists. Ophthalmologist must analyze the evidence supporting the practice of antibiotic prophylaxis and the evidence contraindicating the use of antibiotics, as the overuse of antibiotics could possibly cause the creation and proliferation of resistant strains and increase drug costs and the likelihood of possible adverse reactions to the drugs administered.

This study included the largest meta-analysis published on this subject to date. The results of our study establish that the prophylactic use of antibiotics for intravitreal anti-VEGF injections is associated with a higher incidence of endophthalmitis. This finding could potentially eliminate an unnecessary intervention that is likely harmful to patients. Additionally, we did not observe any benefits associated with the location at which the administration of the intravitreal injection was performed, such as during a consultation at an outpatient clinic as opposed to during surgery or at another location. These two observations have implications for patient comfort, efficiency and the costs of administering these treatments. 


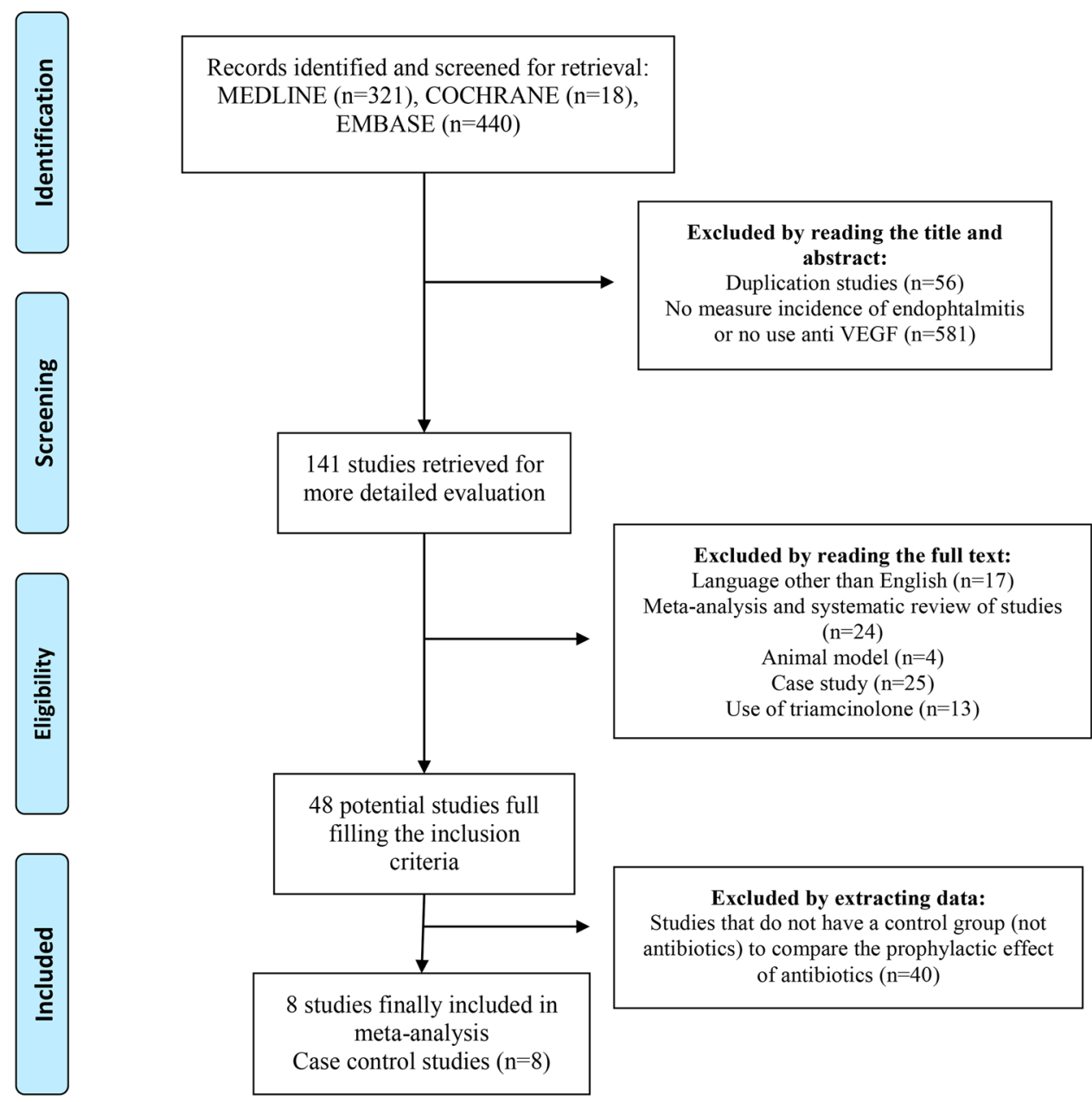

Figure 2. Identification and selection of studies for the meta-analysis.

\section{Materials and Methods}

The Meta-analysis Of Observational Studies in Epidemiology (MOOSE) ${ }^{21}$ criteria along with the Preferred Reporting Items for Systematic Reviews and Meta-Analyses (PRISMA) ${ }^{22}$ were used for this review and meta-analysis (Table S1).

Search methods used to identify studies. We searched MEDLINE, EMBASE and the Cochrane Database of Systematic Reviews from January 2007 (starting date of treatment injecting intravitreal anti-VEGFs) to December 2016. The terms used included "Endophthalmitis" "Antibiotic" and "Intravitreal injection". Different combinations of terms/descriptors were used to ensure the inclusion of a greater number of studies. For details of the search strategy, please see the supplementary materials (Table S2).

Selection criteria for studies. During these investigations, specific predetermined selection criteria were applied to individually assess the eligibility of the studies. The inclusion criteria were as follows: (1) studies published in English, (2) studies that produced original data on the problem and (3) studies examining the incidence, prevalence or risk of post-injection endophthalmitis associated with prophylactic topical antibiotics administered before or after injections of anti-VEGF (ranibizumab, bevacizumab and aflibercept) and prophylactic administration of antibiotics (independent variables).

The exclusion criteria were as follows: (1) studies in which patients were treated with intravitreal anti-VEGF injections in combination with another therapeutic strategy (for example, photodynamic therapy) and (2) studies involving intravitreal triamcinolone injections or another type of steroid used as a main therapeutic agent or combined with other anti-VEGF agents, which is justified because the delimitation only includes a single class of agents, inhibitors of VEGF. We attempted to specifically exclude intravitreal corticosteroids among articles that met the criteria of homogeneity with the rest of the meta-analyses. This process was performed by collaboration of the authors. Table S3 details the PICOS (population, interventions, comparators, outcomes and study.

Study selection, data collection and risk of bias assessment. One author (MB) conducted all literature searches and collated the abstracts. Two authors (MB and RM) separately reviewed the abstracts and determined the suitability of the articles for inclusion in the study based on the selection criteria. Any disagreement 
was resolved through discussions with a third reviewer (AP). In addition, reference lists of all publications meeting the inclusion criteria were manually searched to identify any further studies not identified via electronic searches. The search strategy is described in Fig. 2. To assess the methodological quality of the studies, we used the Strengthening the Reporting of Observational Studies in Epidemiology Statement (STROBE) checklist for observational studies ${ }^{23}$.

Publication bias was assessed graphically using a funnel plot and a Galbraith plot $^{24}$ and via regression tests for funnel plot asymmetry ${ }^{25}$. Identification of publications that could influence the outcome of the study was conducted with the leave-one-out sensitivity analysis ${ }^{26}$.

For quality assessment analysis, the following items were evaluated in each of the publications: (1) Research question explicitly defined, 2) Sample size, 3) Characteristics and definition of study population, 4) Specification of inclusion and exclusion criteria, 5) Good definition and assessment (accurate and reliable methods), 6) Statistical analysis, 7) Completeness of reporting, 8) Study design, 9) External validity of results 10) conflict of interest in the conduct of the study.

These ten items were evaluated for each of the individual studies, assigning a three-level code (1: poor reporting/quality, 2: acceptable, 3: good). The quality score for each of the papers included in the analysis was between acceptable and good quality/reporting.

Data synthesis and analysis. The primary outcome measure was the relative risk (RR) of endophthalmitis. The random effects model with restricted maximum likelihood estimation (Restricted Maximum Likelihood, REML) was used ${ }^{27}$. The existence of statistical heterogeneity between studies was evaluated using the $\mathrm{I}^{2}$ index ${ }^{28,29}$.

A subgroup analysis was also performed to assess the effect of certain covariates on the RR of endophthalmitis. These analyses were conducted using meta-regression ${ }^{30}$ with mixed model effects ${ }^{27}$.

All analyses were performed using the metafor ${ }^{31}$ library in R software (R Core Team, 2016) ${ }^{32}$.

Precis. The use of antibiotics in the prophylaxis of endophthalmitis when administering intravitreal injections of anti-VEGF agents may be harmful. Our meta-regression analysis also indicated that outcomes do not vary with respect to the setting where injection was given (outpatient clinics or operating rooms) of antibiotic administration.

\section{References}

1. Fong, A. H. \& Lai, T. Y. Long-term effectiveness of ranibizumab for age-related macular degeneration and diabetic macular edema. Clinical interventions in aging 8, 467-483, https://doi.org/10.2147/CIA.S36811 (2013).

2. Elman, M. J. et al. Intravitreal Ranibizumab for diabetic macular edema with prompt versus deferred laser treatment: 5-year randomized trial results. Ophthalmology 122, 375-381, https://doi.org/10.1016/j.ophtha.2014.08.047 (2015).

3. Sun, Y. \& Qu, Y. Comparison of intravitreal bevacizumab with intravitreal triamcinolone acetonide for treatment of cystoid macular edema secondary to retinal vein occlusion: a Meta-analysis. International journal of ophthalmology 8, 1234-1239, https://doi. org/10.3980/j.issn.2222-3959.2015.06.29 (2015).

4. Schwartz, S. G. \& Flynn, H. W. Jr. Endophthalmitis Associated with Intravitreal Anti-Vascular Endothelial Growth Factor Injections. Current ophthalmology reports 2, 1-5, https://doi.org/10.1007/s40135-013-0033-1 (2014).

5. Arevalo, J. F., Jap, A., Chee, S. P. \& Zeballos, D. G. Endogenous endophthalmitis in the developing world. International ophthalmology clinics 50, 173-187, https://doi.org/10.1097/IIO.0b013e3181d26dfc (2010).

6. Avery, R. L. et al. Intravitreal injection technique and monitoring: updated guidelines of an expert panel. Retina 34(Suppl 12), S1-S18, https://doi.org/10.1097/IAE.0000000000000399 (2014).

7. Schimel, A. M., Scott, I. U. \& Flynn, H. W. Jr. Endophthalmitis after intravitreal injections: should the use of face masks be the standard of care? Archives of ophthalmology 129, 1607-1609, https://doi.org/10.1001/archophthalmol.2011.370 (2011).

8. Fineman, M. S., Hsu, J., Spirn, M. J. \& Kaiser, R. S. Bimanual assisted eyelid retraction technique for intravitreal injections. Retina 33, 1968-1970, https://doi.org/10.1097/IAE.0b013e318287da92 (2013).

9. Bhavsar, A. R. et al. Update on risk of endophthalmitis after intravitreal drug injections and potential impact of elimination of topical antibiotics. Archives of ophthalmology 130, 809-810, https://doi.org/10.1001/archophthalmol.2012.227 (2012).

10. Storey, P. et al. The role of topical antibiotic prophylaxis to prevent endophthalmitis after intravitreal injection. Ophthalmology 121, 283-289, https://doi.org/10.1016/j.ophtha.2013.08.037 (2014).

11. Samia-Aly, E., Cassels-Brown, A., Morris, D. S., Stancliffe, R. \& Somner, J. E. A survey of UK practice patterns in the delivery of intravitreal injections. Ophthalmic \& physiological optics: the journal of the British College of Ophthalmic Opticians 35, 450-454, https://doi.org/10.1111/opo.12217 (2015).

12. Xing, L., Dorrepaal, S. J. \& Gale, J. Survey of intravitreal injection techniques and treatment protocols among retina specialists in Canada. Canadian journal of ophthalmology. Journal canadien d'ophtalmologie 49, 261-266, https://doi.org/10.1016/j. jcjo.2014.03.009 (2014).

13. Hsu, J., Gerstenblith, A. T., Garg, S. J. \& Vander, J. F. Conjunctival flora antibiotic resistance patterns after serial intravitreal injections without postinjection topical antibiotics. American journal of ophthalmology 157, 514-518 e511, https://doi.org/10.1016/j. ajo.2013.10.003 (2014).

14. Milder, E., Vander, J., Shah, C. \& Garg, S. Changes in antibiotic resistance patterns of conjunctival flora due to repeated use of topical antibiotics after intravitreal injection. Ophthalmology 119, 1420-1424, https://doi.org/10.1016/j.ophtha.2012.01.016 (2012).

15. Kim, S. J. et al. Antibiotic resistance of conjunctiva and nasopharynx evaluation study: a prospective study of patients undergoing intravitreal injections. Ophthalmology 117, 2372-2378, https://doi.org/10.1016/j.ophtha.2010.03.034 (2010).

16. Abell, R. G., Kerr, N. M., Allen, P. \& Vote, B. J. Intravitreal injections: is there benefit for a theatre setting? The British journal of ophthalmology 96, 1474-1478, https://doi.org/10.1136/bjophthalmol-2012-302030 (2012).

17. Tabandeh, H. et al. Endophthalmitis associated with intravitreal injections: office-based setting and operating room setting. Retina 34, 18-23, https://doi.org/10.1097/IAE.0000000000000008 (2014).

18. Benoist d'Azy, C., Pereira, B., Naughton, G., Chiambaretta, F. \& Dutheil, F. Antibioprophylaxis in Prevention of Endophthalmitis in Intravitreal Injection: A Systematic Review and Meta-Analysis. PloS one 11, e0156431, https://doi.org/10.1371/journal.pone.0156431 (2016).

19. VanderBeek, B. L., Bonaffini, S. G. \& Ma, L. The Association between Intravitreal Steroids and Post-Injection Endophthalmitis Rates. Ophthalmology 122, 2311-2315 e2311, https://doi.org/10.1016/j.ophtha.2015.07.005 (2015).

20. Bucher, R. S. et al. Effect of intravitreal triamcinolone acetonide on susceptibility to experimental bacterial endophthalmitis and subsequent response to treatment. Archives of ophthalmology 123, 649-653, https://doi.org/10.1001/archopht.123.5.649 (2005). 
21. Stroup, D. F. et al. Meta-analysis of observational studies in epidemiology: a proposal for reporting. Meta-analysis Of Observational Studies in Epidemiology (MOOSE) group. Jama 283, 2008-2012 (2000).

22. Moher, D., Liberati, A., Tetzlaff, J., Altman, D. G. \& Group, P. Preferred reporting items for systematic reviews and meta-analyses: the PRISMA statement. Journal of clinical epidemiology 62, 1006-1012, https://doi.org/10.1016/j.jclinepi.2009.06.005 (2009).

23. Vandenbroucke, J. P. et al. Strengthening the Reporting of Observational Studies in Epidemiology (STROBE): explanation and elaboration. Epidemiology 18, 805-835, https://doi.org/10.1097/EDE.0b013e3181577511 (2007).

24. Galbraith, R. F. Some Applications of Radial Plots. Journal of the American Statistical Association 89, 1232-1242, https://doi. org/10.2307/2290987 (1994).

25. Harbord, R. M., Egger, M. \& Sterne, J. A. A modified test for small-study effects in meta-analyses of controlled trials with binary endpoints. Statistics in medicine 25, 3443-3457, https://doi.org/10.1002/sim.2380 (2006).

26. Viechtbauer, W. \& Cheung, M. W. Outlier and influence diagnostics for meta-analysis. Research synthesis methods 1, 112-125, https://doi.org/10.1002/jrsm.11 (2010).

27. Raudenbush, S. W. The handbook of research synthesis and meta-analysis. (eds. Cooper, H. et al.). Ch. 16, pp. 295-315 (New York: Russell Sage Foundation, 2009).

28. Higgins, J. P. \& Thompson, S. G. Quantifying heterogeneity in a meta-analysis. Statistics in medicine 21, 1539-1558, https://doi. org/10.1002/sim.1186 (2002).

29. Higgins, J. P., Thompson, S. G., Deeks, J. J. \& Altman, D. G. Measuring inconsistency in meta-analyses. Bmj 327, 557-560, https:// doi.org/10.1136/bmj.327.7414.557 (2003).

30. Thompson, S. G. \& Higgins, J. P. T. How should meta-regression analyses be undertaken and interpreted? Statistics in medicine 21, 1559-1573, https://doi.org/10.1002/sim.1187 (2002).

31. Viechtbauer, W. Conducting Meta-Analyses in R with the metafor Package. 2010 36, 48, https://doi.org/10.18637/jss.v036.i03 (2010).

32. Team, R. C. R: A language and environment for statistical computing. R Foundation for Statistical Computing, Vienna, Austria. 2015. http.www.R-project.org (2016).

33. Falavarjani, K. G. et al. Incidence of acute endophthalmitis after intravitreal bevacizumab injection in a single clinical center. Retina 33, 971-974, https://doi.org/10.1097/IAE.0b013e31826f0675 (2013).

34. Cheung, C. S. et al. Incidence of endophthalmitis and use of antibiotic prophylaxis after intravitreal injections. Ophthalmology 119, 1609-1614, https://doi.org/10.1016/j.ophtha.2012.02.014 (2012).

35. Mason, J. O. 3rd et al. Incidence of acute onset endophthalmitis following intravitreal bevacizumab (Avastin) injection. Retina 28, 564-567, https://doi.org/10.1097/IAE.0b013e3181633fee (2008).

36. Park, Y., Kim, K. S. \& Park, Y. H. Acute endophthalmitis after intravitreal injection and preventive effect of preoperative topical antibiotics. Journal of ocular pharmacology and therapeutics: the official journal of the Association for Ocular Pharmacology and Therapeutics 29, 900-905, https://doi.org/10.1089/jop.2013.0052 (2013).

37. Falavarjani, K. G. et al. Endophthalmitis after resident-performed intravitreal bevacizumab injection. Canadian journal of ophthalmology. Journal canadien d'ophtalmologie 50,33-36, https://doi.org/10.1016/j.jcjo.2014.09.005 (2015).

38. Storey, P. et al. The effect of prophylactic topical antibiotics on bacterial resistance patterns in endophthalmitis following intravitreal injection. Graefe's archive for clinical and experimental ophthalmology = Albrecht von Graefes Archiv fur klinische und experimentelle Ophthalmologie 254, 235-242, https://doi.org/10.1007/s00417-015-3035-x (2016).

39. Li, A. L. et al. Endophthalmitis after intravitreal injection: role of prophylactic topical ophthalmic antibiotics. Retina 36, 1349-1356, https://doi.org/10.1097/IAE.0000000000000901 (2016).

\section{Acknowledgements}

We thank the authors who collaborated in the compilation of this article by providing additional information. We also thank the statistical center Biostatech (Universidad de Santiago de Compostela, Spain) for providing the averages for the preparation of this work.

\section{Author Contributions}

Acquisition of data: M.B., R.M. and A.P. Analysis and interpretation of data: M.P. and M.B. Drafting of manuscript: M.B. and F.G.U. Critical revision: M.F. and M.B.-T. All authors reviewed the manuscript.

\section{Additional Information}

Supplementary information accompanies this paper at https://doi.org/10.1038/s41598-017-18412-9.

Competing Interests: The authors declare that they have no competing interests.

Publisher's note: Springer Nature remains neutral with regard to jurisdictional claims in published maps and institutional affiliations.

Open Access This article is licensed under a Creative Commons Attribution 4.0 International License, which permits use, sharing, adaptation, distribution and reproduction in any medium or format, as long as you give appropriate credit to the original author(s) and the source, provide a link to the Creative Commons license, and indicate if changes were made. The images or other third party material in this article are included in the article's Creative Commons license, unless indicated otherwise in a credit line to the material. If material is not included in the article's Creative Commons license and your intended use is not permitted by statutory regulation or exceeds the permitted use, you will need to obtain permission directly from the copyright holder. To view a copy of this license, visit http://creativecommons.org/licenses/by/4.0/.

(c) The Author(s) 2017 